\title{
CHANGEMENT DES POLITIQUES NATIONALES D'ACQUISITION D'ARMEMENT ET COOPÉRATION EUROPÉENNE.
}

Le cas des programmes d'hélicoptère tigre et de missiles trigat.

Catherine Hoeffler

\section{L'Harmattan | « Politique européenne »}

$2008 / 3 n^{\circ} 26 \mid$ pages 211 à 220

ISSN 1623-6297

ISBN 9782296074866

Article disponible en ligne à l'adresse :

http://www.cairn.info/revue-politique-europeenne-2008-3-page-211.htm

\section{Pour citer cet article :}

Catherine Hoeffler, « Changement des politiques nationales d'acquisition d'armement et coopération européenne. Le cas des programmes d'hélicoptère tigre et de missiles trigat. », Politique européenne 2008/3 ( $\mathrm{n}^{\circ}$ 26), p. 211-220. DOI 10.3917/poeu.026.0211

Distribution électronique Cairn.info pour L'Harmattan.

(C) L'Harmattan. Tous droits réservés pour tous pays.

La reproduction ou représentation de cet article, notamment par photocopie, n'est autorisée que dans les limites des conditions générales d'utilisation du site ou, le cas échéant, des conditions générales de la licence souscrite par votre établissement. Toute autre reproduction ou représentation, en tout ou partie, sous quelque forme et de quelque manière que ce soit, est interdite sauf accord préalable et écrit de l'éditeur, en dehors des cas prévus par la législation en vigueur en France. Il est précisé que son stockage dans une base de données est également interdit. 


\section{CHANGEMENT DES POLITIQUES NATIONALES D'ACQUISITION D'ARMEMENT ET COOPERATION EUROPEENNE. LE CAS DES PROGRAMMES D'HELICOPTERE TIGRE ET DE MISSILES TRIGAT.}

\section{La coopération européenne en matière d'armement}

Dès le début de la guerre froide, la coopération européenne en matière d'armement a été prônée pour répondre à des buts tant militaires (répondre à la menace soviétique, renforcer l'interopérabilité), que budgétaires (mutualiser les coûts des politiques) ou industriels (soutenir les tissus industriels de défense nationaux). Elle se retrouve à différents niveaux : international avec l'OTAN, européen avec l'Union de l'Europe Occidentale et bilatéral avec des accords politiques intergouvernementaux (tels que le Traité de l'Elysée). Plus spécifiquement, la coopération s'incarne dans la mise en place de programmes d'armement en coopération, représentant l'harmonisation effective des politiques, puisque plusieurs Etats s'engagent conjointement, avec leurs industries nationales respectives, dans la production et l'achat d'un même armement. La promotion de la coopération a été renforcée depuis ces vingt dernières années comme un moyen de répondre aux changements internationaux (fin de la guerre froide) et domestiques (réduction des budgets): les restructurations et intégrations des groupes en entités européennes sont encouragées comme le moyen de rationaliser un paysage industriel trop morcelé, donc trop vulnérable face à une concurrence mondiale accrue et une baisse structurelle des budgets nationaux ne permettant plus aux Etats de supporter seuls le maintien du tissu industriel. En d'autres termes, il s'agit d'harmoniser l'offre aussi bien que la demande d'un marché européen de l'armement, seul capable, grâce à sa taille et aux économies d'échelle qu'il offrirait, d'assurer la survie des industries de défense, et de là, l'autonomie d'approvisionnement des Etats..

Pourtant, si l'intégration des industries de défense est partiellement atteinte avec la création de la maison mère EADS et de

politique européenne $\mathrm{n}^{\circ} 26$, automne 2008, p.211-220 
ses différentes filiales telles que Eurocopter dans les hélicoptères ou MBDA $^{1}$ dans les missiles ainsi que par la consolidation de groupes tels que BAE Systems ou Thalès, l'on peut s'interroger sur les mécanismes et résultats de la coopération au niveau des politiques d'armement. En l'absence d'autorité européenne en la matière, quels sont les mécanismes de leur harmonisation? S'agit-il d'une européanisation ou d'une simple coopération? Quels sont les facteurs influençant le succès ou l'échec de cette harmonisation au sein des programmes d'armement? En d'autres termes : comment change la coopération européenne en matière d'armement?

\section{Le puzzle empirique : à coopérations comparables, des évolutions différentes}

Le segment des armements antichar, avec comme cas d'étude les programmes d'hélicoptère (Tigre) et de missiles (Trigat) offre un cadre d'analyse pertinent pour analyser la coopération européenne en matière d'armement: (1) ces programmes montrent une similitude dans les inputs: nés dans un même contexte (années 1970), ils rassemblent la France, l'Allemagne et le Royaume-Uni², répondent au même besoin opérationnel et ont des structures industrielles et institutionnelles comparables ${ }^{3}$; (2) il s'agit de domaines avec une coopération de longue date, marquée par le succès, notamment pour les missiles ; (3) ils sont tous deux touchés par les changements domestiques des années 1990 et par le choc exogène que constitue la

${ }^{1}$ European Aeronautic Defence and Space company (EADS) ; Matra BAe Dynamics Alenia (MBDA).

${ }^{2}$ Le Royaume-Uni ne participe pas au programme Tigre mais sa position est tout de même à prendre en compte dans l'évolution du programme : outre son besoin en hélicoptères, son implication au sein du Trigat, missiles complémentaires du Tigre, peut être lue comme une incitation industrielle et politique en faveur du Tigre.

${ }^{3}$ D’un point de vue politico-administratif, la coopération est gérée par un bureau de programme transnational; les industries coopèrent tout d'abord via un groupement d'intérêt économique (GIE) et enfin, dans le cas des hélicoptères, par la fusion des filiales hélicoptéristes au sein de l'entreprise Eurocopter.

${ }^{4}$ La génération de missiles précédente (Hot et Milan) avait représenté un succès tant militaire, technologique que commercial, le GIE franco-allemand Euromissile devenant leader mondial de ce segment d'armement. 
fin de la guerre froide et qui représente une menace forte à leur "survie », ces programmes étant destinés à un scénario d'engagement typique de ce conflit bipolaire; (4) pourtant, malgré une apparente institutionnalisation de la coopération dans ce secteur, avec une offre et une demande a priori harmonisée, les réponses nationales divergent finalement quant à l'avenir de ces deux programmes : le RoyaumeUni abandonne les deux programmes et achète des équipements américains ; la France maintient et opère une conversion du Tigre aux nouveaux besoins, mais abandonne le Trigat, arguant de son inadaptation aux nouveaux conflits, et achète des missiles étrangers ; l'Allemagne maintient les deux programmes; (5) le résultat de la coopération montre: une "désharmonisation» européenne dans le cas du Trigat, avec l'achat de missiles étrangers par de nombreux pays; une harmonisation partielle dans le cas du Tigre, avec l'existence de deux versions françaises et allemandes très différentes.

Notre étude nous permet donc d'analyser les mécanismes (les programmes) et les résultats différenciés (maintien, abandon, conversion des politiques) de la coopération européenne en matière d'armement. Cette analyse éclaire en outre plus largement les modalités de l'édification d'une Europe de la défense, compte tenu de l'importance que recouvre l'harmonisation des armements nationaux dans ce projet.

\section{Positionnement théorique et hypothèses de recherche}

Dans une perspective croisant le néo-institutionnalisme historique 5 appliqué à l'étude des politiques publiques et l'économie politique internationale ${ }^{6}$, nous expliquons l'évolution de la

5 Pierson P., 1996, «The Path to European Integration. A Historical Institutionalist Analysis ", Comparative Political Studies, vol. 29, No. 2, pp. 123-163; Pierson P., Skocpol T., 2002, "Historical Institutionalism in Contemporary Political Science", in Katznelson I., Milner H. (eds..), Political science: state of the discipline, New York, Norton, pp. 693-721

${ }_{6}$ Gourevitch P., Politics in Hard Times. Comparative Responses to International Economic Crisis, Ithaca, Cornell University Press, 1986; Thatcher M., 1999, The Politics of Telecommunications. National Institutions, Convergence, and Change in Britain and France, Oxford, Oxford University Press ; Fligstein N., The architecture of markets: an economic sociology of twenty-first century capitalist societies, Princeton, Princeton University Press, 2001; Fligstein N., «The Structural Transformation of American Industry: An Institutional Account of the 
coopération européenne par le changement des politiques nationales d'armement? ${ }^{7}$ Nous reprenons d'abord notre positionnement théorique avant d'énoncer nos hypothèses d'analyse.

L'étude des programmes d'armement a donné naissance à une littérature académique spécifique, très développée aux Etats-Unis ${ }^{8}$ et connaissant un renouveau dans la recherche française ${ }^{9}$. Le changement apparait pourtant comme un angle mort de ces études, question pourtant cruciale pour nos cas. En effet, dans des perspectives très différentes, les analyses soulignent majoritairement l'inertie des politiques ou le maintien des programmes d'armement, ce malgré un choc exogène comme la fin de la guerre froide ou leur déconnection de besoins militaires ${ }^{10}$. La persistance de ces politiques est tour à tour expliquée par une logique technologique propre, l'intérêt d'acteurs organisés (complexe militaro-industriel) ou encore la création d'une coalition fédérée autour d'une croyance légitimant le maintien du programme. Outre l'accent mis sur les acteurs, ces analyses se concentrent sur les programmes d'armement, lieu et processus traditionnels de la réalisation effective des politiques d'armement. Cependant, cette focalisation comporte ses limites:

Causes of Diversification in the Largest Firms, 1919-1979», in Powell W. W., DiMaggio, P. J. (dir), The New Institutionalism in Organizational Analysis, The University of Chicago Press, Chicago and London, 1991, p. 311-336.

7 Muller P., 2005, «Esquisse d'un théorie du changement dans l'action publique. Structures, acteurs et cadres cognitifs », Revue française de science politique, vol. 55 (1), p. 155-187 ; Hassenteufel P., 2005, «De la comparaison internationale à la comparaison transnationale. Les déplacements de la construction d'objets comparatifs en matière de politiques publiques ", Revue française de science politique, vol. 55 (1), p.113-132.

8 Les paradigmes classiques d'analyses sont ceux du complexe militaroindustriel, du paradigme bureaucratique et des dynamiques technologiques portées par les ingénieurs, dont respectivement: Mills C. W., L'Elite du pouvoir, Paris, Maspero, 1969 ; Allison G., Essence of Decision. Explaining the Cuban Missile Crisis, Boston, Little Brown, 1971; Kaldor M., The Baroque Arsenal, Londres, A. Deutsch, 1982.

9 Joana J., Smith A., "Changing French Military Procurement Policy: The State, Industry and 'Europe' in the Case of the A400M", West European Politics, vol. 29, No. 1, janvier 2006 ; Genieys W. (dir.), Le choix des armes. Théories, acteurs et politiques, Paris, CNRS Ed., 2004 ; Genieys W., Michel L., "Au-delà du complexe militaro-industriel. Le rôle d'une élite sectorielle dans le programme du char Leclerc », Revue française de sociologie, 47-1, 2006.

10 Respectivement dans: W. Genieys et L. Michel, op. cit. ; Mary Kaldor, op. cit. 
d'une part, peu d'analyses portent sur des coopérations internationales, alors même que cette échelle est devenue incontournable; d'autre part, cette focalisation induit un biais en faveur d'une lecture dichotomique entre réussite et échec, incluant par là même une lecture en termes d'intérêt et un accent trop accru sur ce qui n'est en réalité qu'une forme historiquement prise par la coopération et en constante évolution.

Notre analyse entend ainsi replacer l'étude des programmes d'armement dans le cadre de l'analyse des institutions des politiques nationales d'acquisition d'armement. La coopération européenne par programmes est forgée tout à la fois par une logique interne - un processus endogène d'apprentissage - et par l'évolution des buts et instruments des politiques nationales. Il convient donc de prendre en compte dans un premier temps les logiques endogènes d'acteurs pour mieux montrer que la compréhension du devenir des programmes dépend de l'adéquation des stratégies de ces acteurs impliqués avec les logiques institutionnelles nationales.

W. Genieys et L. Michel ont expliqué le maintien d'un programme par le succès d'une coalition d'acteurs fédérés autour d'une croyance commune permettant de légitimer et de pérenniser le programme ${ }^{11}$. Le cas du Trigat montre a contrario que la supposée coalition s'est dissoute, avec la défection des acteurs britanniques et français. L'explication endogène ne donne pas de piste pour expliquer ce changement des représentations ou intérêts des acteurs au sein de la coalition. Il est donc nécessaire de déplacer le regard pour comprendre les conditions d'existence d'une telle coalition ${ }^{12}$ et de là,

11 W. Genieys et L. Michel, op. cit.

12 Nous nous référons ici au débat traditionnel entre structure et agent au sein de l'institutionnalisme, cf. Beckert J. «Agency, Entrepreneurs, and Institutional Change. The Role of Strategic Choice and Institutionalized Practices in Organizations », Organization Studies, Vol. 20, No. 5, 1999, p. 777-799. Si nous ne réfutons pas une capacité d'innovation des «acteurs socialement doués » de Neil Fligstein, il semble toutefois que l'intérêt même de ces derniers soient largement façonné et encadré par des changements insitutionnels qui lui préexistent, cf. : Grossman E. «Bringing politics back in: rethinking the role of economic interest groups in European integration ", Journal of European Public Policy, vol. 11, no 4, 2004, p. 637654 »; Woll C., Firm Interets: How Governments Shape Business Lobbying on Global Trade. Ithaca, N.Y., Cornell University Press, 2008. S’il n'est pas aisé de démontrer un impact causal empirique systématique, nous montrerons comment les institutions ont forgé les identités organisationnelles et stratégies des acteurs. Cette articulation entre institutions et acteurs devra 
de survie d'un programme. Or c'est bien le changement institutionnel national qui explique le destin d'un programme en coopération.

En ce sens, ces deux programmes ont fonctionné comme des « consensus ambigus "13 $^{13}$ entre acteurs nationaux répondant à des évolutions institutionnelles propres. Les deux programmes répondaient en réalité de manière contrastée aux nouveaux critères de légitimité des politiques nationales : ces critères ont été redéfinis par le réagencement des équilibres institutionnels entre logiques politiques, militaires, industrielle et de marché forgeant les politiques d'armement. En l'état actuel de nos recherches, la variable déterminante est l'équilibre trouvé entre logique de marché et logique industrielle : cet équilibre varie selon les pays et les types d'armement. L'évolution de la posture de l'Etat dans la régulation du marché joue donc un rôle fondamental pour expliquer tant la temporalité, les mécanismes et les résultats de la coopération européenne au sein de ces deux programmes.

Ceci permet ici de franchir une troisième étape d'analyse en analysant la mise en œuvre de cette nouvelle hiérarchisation des buts d'une politique d'armement et, de là, les conditions et les formes prises par la coopération européenne. Nous questionnons par là la notion d'européanisation ${ }^{14}$, en montrant qu'elle ne peut qu'être imparfaitement adaptée à l'évolution de la coopération européenne en matière d'armement.

Trois hypothèses de recherche se dégagent :

1/ Depuis la guerre froide, la coopération en matière d'armement s'est organisée autour de programmes. C'est en effet par leur biais que les acteurs nationaux, politiques, administratifs, militaires et

faire l'objet d'un développement théorique et méthodologique plus ample et rigoureux.

${ }_{13}$ Sur la notion de consensus ambigu, voir: Palier B., 2003, « Gouverner le changement des politiques de protection sociale », in Favre P., Hayward J., Schemeil Y., Etre gouverné. Mélanges offerts en l'honneur de Jean Leca, Paris, Presses de Sciences Po, p. 163-179. Voir aussi: Jabko N., 2006, Playing the Market: A Political Strategy for Uniting Europe, 1985-2005, Ithaca, Cornell University press.

${ }^{14}$ Radaelli C., 2005, «Diffusion without convergence: how political context shapes the adoption of regulatory impact assessment ", Journal of European Public Policy, vol. 12 n5, pp. 924-943; Palier B., Surel Y. et al, 2007, L’Europe en action: l'européanisation dans une perspective comparée, Paris, L'Harmattan ; Jacquot S., Woll C. (dir.), 2004, Les usages de l'Europe: acteurs et transformations européennes, Paris, L'Harmattan, 2004. 
industriels s'organisent: sont créées au niveau politique et administratif des instances de coopération, et au niveau industriel des alliances de plus en plus intégrées entre firmes nationales. Pourtant, l'apparente institutionnalisation de la coopération donne à voir des évolutions incrémentales aux niveaux nationaux vers l'affirmation de divergences quant aux buts nationaux poursuivis au sein de la coopération. Ces changements incrémentaux sont à observer tant au niveau national qu'au niveau proprement dit du programme qui les répercute et les incarne. Ils restent pourtant invisibles, la coopération étant assurée par la stabilisation des logiques institutionnelles militaires (une menace commune) et politiques (une volonté de coopération).

2/ Les années 1990, marquées par le changement de la menace et la redéfinition de l'action de l'Etat dans le secteur, représentent le moment d'une divergence des politiques nationales d'armement, avec pour conséquence l'échec total ou partiel de l'harmonisation via les programmes.

Cette période, marquée par l'incertitude, a représenté une fenêtre d'opportunité saisie et construite par certains acteurs pour redéfinir les buts et instruments légitimes d'une politique d'acquisition. Compte tenu des réponses nationales fort différentes au choc exogène que constitue la fin de la Guerre froide, il est peu heuristique de s'arrêter à cette dernière comme cause ultime des changements domestiques. Au contraire, ce choc a été largement façonné par les prismes institutionnels nationaux et leurs évolutions portant sur le but des politiques d'armement et sur le rôle que doit jouer l'Etat dans la régulation du secteur.

La variété des positions nationales (achat sur étagère, maintien en coopération ou maintien national) s'éclaire au vu de ces changements. Les trois pays présentent en ce sens une divergence dans leurs évolutions institutionnelles. La fin de la guerre froide a joué le rôle de révélateur de divergences latentes dans les coopérations déjà existantes et, enfin, celui de catalyseur d'affirmation de ces divergences. Concrètement, il apparait qu'en France les changements des années 1990 ont représenté l'opportunité de redéfinir la place de l'Etat dans la régulation du secteur vers un modèle plus libéral inspiré du système britannique, ces évolutions ont au contraire signifié le retour d'une politique industrielle de défense allemande, impossible à imaginer avant la réunification et la réhabilitation militaire 
allemande ${ }^{15}$. Cette divergence est claire dans un cas considéré comme non stratégique (Trigat). Au contraire, elle a été partiellement neutralisée dans le cas du Tigre de deux manières : par la politisation du programme qui assura son maintien; la possibilité pour l'Etat arbitre ou régulateur de passer outre les règles de marché quand il s'agit de secteurs stratégiques. En ce sens, l'hélicoptère Tigre représente dans le cas de la France un cas de conversion de politique d'armement, d'une application antichar à celle d'appui combat.

Cette approche pourra s'insérer plus largement dans une réflexion sur les mécanismes et résultats d'une libéralisation ${ }^{16}$ du secteur de l'armement; il conviendra d'étudier à ce titre les acteurs promoteurs de ce mouvement, même si l'analyse systématique de ces derniers est difficile dans ce secteur. Cette approche analytique permet ainsi de comprendre la combinaison de positions aussi différentes que le maintien des politiques industrielles et la promotion de logiques de marché. En effet, les études sur la libéralisation nous ont appris que cette dernière ne représentait en rien un affaiblissement des capacités étatiques - en ce sens, la libéralisation du marché de l'armement ne signifie pas que l'Etat ne puisse pas décider, contre la logique prévalente de marché, d'affirmer une politique industrielle, comme ce fut le cas avec le Trigat en Allemagne.

3/ Enfin, nous revenons à l'échelle de la coopération européenne et faisons l'hypothèse que son évolution ne s'explique pas par une européanisation mais par des tentatives d'institutionnalisation successives d'un équilibre entre logique de marché et logique industrielle à l'échelle européenne.

La notion d'européanisation ne semble pas heuristique pour rendre compte de l'évolution de la coopération européenne en matière d'armement. Que ce soit dans un sens «dur»

15 Maneval H., 1994, "Country Survey III. Defence Spending in West Germany", Defence and Peace Economics, vol. 5, pp. 221-246; Rohde J., Schmidt P., 1995, "German Armaments Policy : its Consequences for the Armaments Industry", Defense Analysis, Vol. 11, No. 3, pp. 269-277; Young T.-D., 1995, "Nationalization or Integration? The Future Direction of German Defense Policy", Defense Analysis, Vol. 11 No 2, p. 109-120.

${ }^{16}$ Nous n'affirmons pas que ce marché a été libéralisé tel un autre marché civil; il a connu pourtant une évolution dans cette direction, avec la privatisation de la majorité des entreprises, la réforme des politiques d'acquisition sur un modèle de nouveau management public et le développement d'outils contractuels visant à une meilleure rentabilité de l'action publique, et l'ouverture de certains marchés à la concurrence. 
d'harmonisation ou plus souple de partage ou diffusion cognitive, ce concept ne permet pas d'analyser les décisions nationales quant au devenir des programmes en coopération. En effet, tout d'abord, l'harmonisation n'est réellement atteinte dans aucun de nos cas. Ensuite, même la convergence des modèles d'acquisition nationaux sur un modèle néomanagérial ne présume pas de l'orientation des décisions politiques en faveur d'une logique de marché ou d'une logique industrielle : l'Etat reste capable d'outrepasser ces règles dans le cas d'un secteur stratégique. En ce sens, si elle existe au niveau des discours politiques et des instruments d'acquisition d'armement, l'européanisation reste muette quant aux résultats effectifs de la coopération.

La coopération européenne et les formes qu'elle prend (programme, organismes européens, renforcement du pilier défense de l'Union européenne) sont en réalité façonnées par les tensions institutionnelles nationales entre logique de marché et logique industrielle. Or si la coopération par programme pouvait promettre une réconciliation de ces deux objectifs (acheter moins cher et soutenir les industries), nos deux cas d'étude montrent qu'il n'en est rien. Ces tensions ont justement mené à un relatif échec de l'harmonisation des armements au sein des programmes. L'expérience des programmes et l'évolution institutionnelle des pays ont montré que logique de marché et logique industrielle ne se combinent pas de manière évidente ou immédiate au niveau européen : acquérir des armements européens en coopération n'est nullement gage de respect d'une logique de marché et de rentabilité de l'action publique; réciproquement, respecter les règles de la concurrence et chercher une efficacité accrue des dépenses publiques peut impliquer d'abandonner à terme certaines compétences industrielles européennes.

En outre, notre lecture est validée par les évolutions européennes dans la régulation de ce secteur. Il n'est à ce titre pas étonnant que les efforts en vue de l'harmonisation se portent essentiellement sur les instruments à la fois de rationalisation des programmes d'armement et d'harmonisation des besoins militaires. Le premier type d'instrument permet de contraindre les politiques nationales de soutien industriel ; le développement du second type peut être interprété comme une tentative renouvelée de recréer une «menace commune» européenne, seul facteur de stabilisation de la coopération par le passé. On observe à ce titre la création de 
l'OCCAR ${ }^{17}$ et de l'Agence Européenne de Défense (AED). Si la première est essentiellement gestionnaire, la dernière a en effet pour but de permettre une meilleure harmonisation des besoins militaires nationaux. Malgré ses faibles ressources et sa dépendance vis-à-vis des Etats, sa mise en place peut être considérée comme une tentative de faire converger en amont les exigences nationales afin d'éviter les écueils possibles de la coopération par programme. Par ailleurs, l'implication de la Commission européenne dans les affaires d'armement via la régulation des marchés publics est intéressante à analyser comme les prémisses d'une européanisation "par le haut», tendant à faire converger les instruments des politiques d'armement par le biais du levier institutionnel potentiellement fort que constituent les règles de la concurrence et le projet d'établissement d'un marché européen de l'armement.

\section{Méthodologie et terrain de recherche}

Notre thèse s'appuie sur un travail de recherche documentaire et d'entretiens. Concernant l'analyse de l'évolution institutionnelle des politiques d'acquisition d'armement dans les trois pays, nous nous appuyons sur la littérature en science politique ainsi que sur la documentation primaire existante sur le sujet. La recherche documentaire concernant l'évolution des programmes repose quant à elle sur trois sources : une revue de presse exhaustive, incluant presse généraliste et spécialisée (source Factiva) ; le dépouillement des archives parlementaires; la littérature sectorielle portant sur l'évolution des armements et des technologies. Enfin, nous conduisons une série d'entretiens semi-directifs auprès de représentants politiques, administratifs, militaires et industriels en lien avec la politique d'armement en général, et plus spécifiquement ces deux programmes, au sein des trois pays.

17 Organisation de Coopération Conjointe en matière d'Armement. 